\title{
Infarto agudo al miocardio asociado al uso de cocaína
}

\section{Acute myocardial infarction associated with cocaine use}

\author{
Natalia Córdoba-Pulido, Nelson L. Moreno-Ruiz • Bogotá, D.C. (Colombia)
}

DOI: https://doi.org/10.36104/amc.2020.1642

\section{Resumen}

El abuso de cocaína se asocia con un mayor riesgo de eventos cardiovasculares, como infarto de miocardio y accidente cerebrovascular isquémico. Los mecanismos fisiopatológicos no se entienden completamente, aunque la formación de trombos intravasculares y la aterosclerosis acelerada son hallazgos destacados. Reportamos el caso de un hombre de 38 años adicto a la cocaína, que presentó eventos isquémicos caracterizados por infarto agudo de miocardio complicado por insuficiencia cardíaca. Se discute la fisiopatología del daño vascular inducido por la cocaína y el manejo de las complicaciones. (Acta Med Colomb 2020; 45. DOI: https://doi.org/10.36104/amc.2020.1642).

Palabras clave: cocaína, endotelio vascular, infarto de miocardio.

\section{Abstract}

Cocaine abuse is related to a greater risk of cardiovascular events such as myocardial infarction and ischemic cerebrovascular accidents. The pathophysiological mechanisms are not fully understood, although the formation of intravascular thrombi and accelerated atherosclerosis are notable findings. We report the case of a 38 -year-old man addicted to cocaine who presented ischemic events in the form of acute myocardial infarction complicated by heart failure. The pathophysiology of cocaine-induced vascular damage and the treatment of complications are discussed. (Acta Med Colomb 2020; 45. DOI: https://doi.org/10.36104/amc.2020.1642).

Key words: cocaine, vascular endothelium, myocardial infarction.
Dra. Natalia Córdoba-Pulido: Residente II año de Medicina Interna, Facultad de Medicina, Fundación Universitaria Sanitas; Dr. Nelson L. Moreno-Ruiz: Médico Cardiólogo, Clínica Universitaria Colombia. Bogotá, D.C. (Colombia). Correspondencia: Dra. Natalia Cordoba-Pulido. Bogotá, D.C. (Colombia)

E-mail: nataliacordoba2006@gmail.com

Recibido: 07/I/2020 Aceptado: 27/V/2020

\section{Introducción}

La cocaína es la segunda droga ilícita más utilizada en Colombia, sólo superada por la marihuana, con una prevalencia de consumo para el 2017 de $3.23 \%$. En cuanto a distribución geográfica Antioquia y los departamentos del Eje Cafetero tienen consumos de drogas ilícitas superiores al resto del país y se presenta más en hombres que en mujeres con mayor consumo entre los 18 y 24 años de edad (1).

Hasta la fecha no se ha registrado a nivel nacional estimación de las visitas a salas de emergencia relacionadas con el consumo de sustancias psicoactivas (SPA). Se han realizado estudios aislados en Bogotá que estiman que ocho de cada cien personas que consultan a urgencias han consumido alguna SPA en las últimas seis horas (2).

La incidencia reportada de infarto de miocardio en pacientes con dolor torácico asociado a cocaína oscila entre 0.7 y $5.7 \%(3,4,5)$ y parece ser un contribuyente importante para IAM entre los jóvenes.

Se presenta un caso de un infarto agudo al miocardio asociado al uso de cocaína en un paciente joven con adicción a ésta.

\section{Caso clínico}

Se presenta a un paciente de sexo masculino de 38 años de edad, quien ingresa al servicio de emergencia por cuadro clínico de tres días de evolución, consistente en dolor torácico retroesternal tipo picada, desencadenado con el esfuerzo físico asociado a disnea y náuseas, único antecedente de importancia refiere consumo de cocaína inhalada desde hace 10 años y hábito enólico desde el mismo tiempo, sin otros hallazgos en la revisión por sistemas.

En el examen físico se encontró un paciente en adecuadas 
condiciones generales con signos vitales: tensión arterial $127 / 85 \mathrm{~mm} / \mathrm{Hg}$, frecuencia cardiaca de $95 \mathrm{LPM}$, frecuencia respiratoria de 21 RPM, oximetría $97 \%$ sin requerimientos de oxígeno suplementario, resto de sistemas sin hallazgos.

Se toma electrocardiograma de 12 derivaciones encontrando lesión subepicárdica en cara anterior extensa (Figura 1), configurando un infarto agudo de miocardio (IAM) con elevación del segmento s-t, a las dos horas es llevado a angioplastia primaria encontrando obstrucción de $100 \%$ de la arteria descendente anterior en el tercio proximal (Figura 2A), lugar donde se realiza implante de dos stent medicados (Figura 2B-2C)
El paciente evoluciona adecuadamente sin complicaciones mecánicas o eléctricas del infarto, sin nuevos episodios de dolor torácico, con ecocardiograma que evidencia cardiopatía isquémica con fracción de eyección del ventrículo izquierdo de $30 \%$, por lo que se inicia bloqueo neurohumoral con adecuada tolerabilidad, se completa primera fase de rehabilitación cardiovascular y posterior a valoración por psiquiatría e inclusión al programa de control de adicciones se da egreso en el día cuatro postinfarto, para completar manejo ambulatorio por un año con antiagregación dual ASA + prasugrel, sin otras complicaciones.

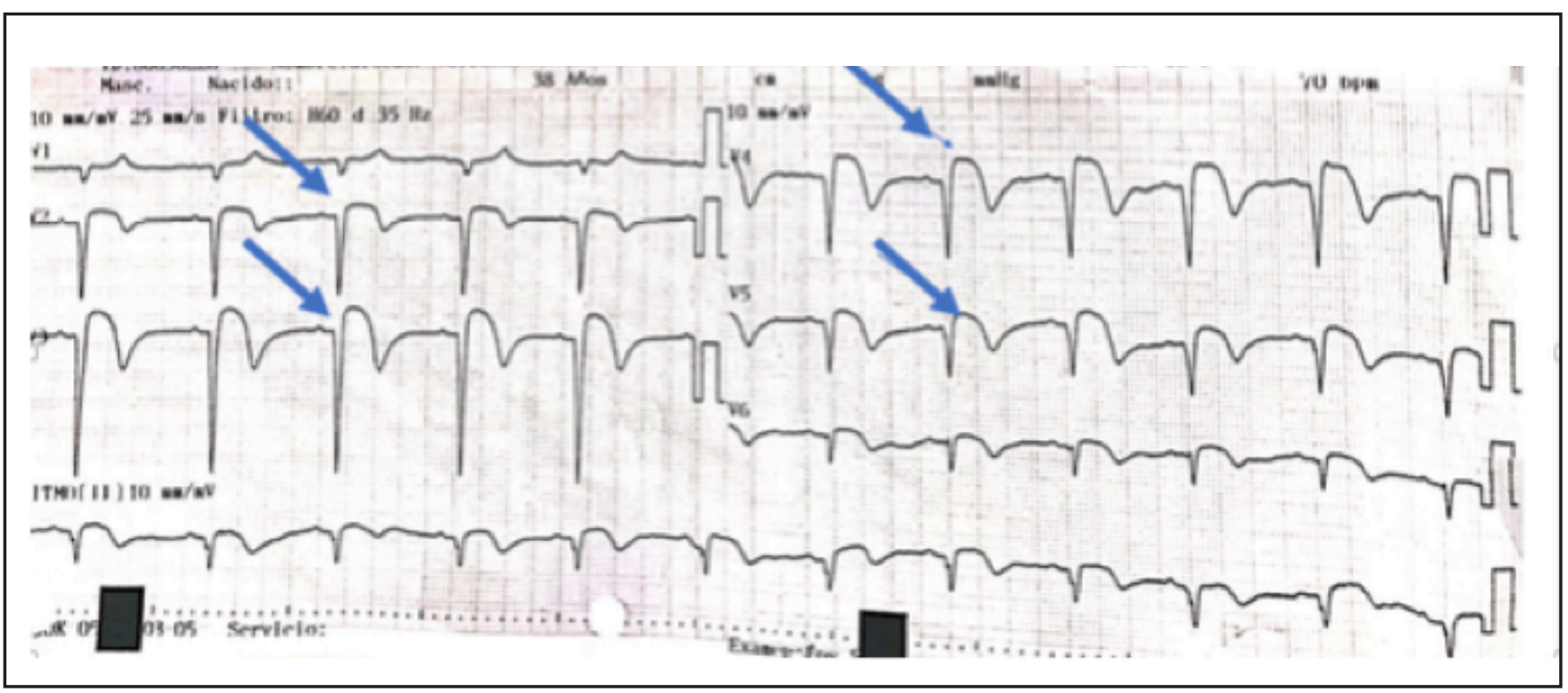

Figura 1. Electrocardiograma lesión subepicárdica en cara anterior extensa.

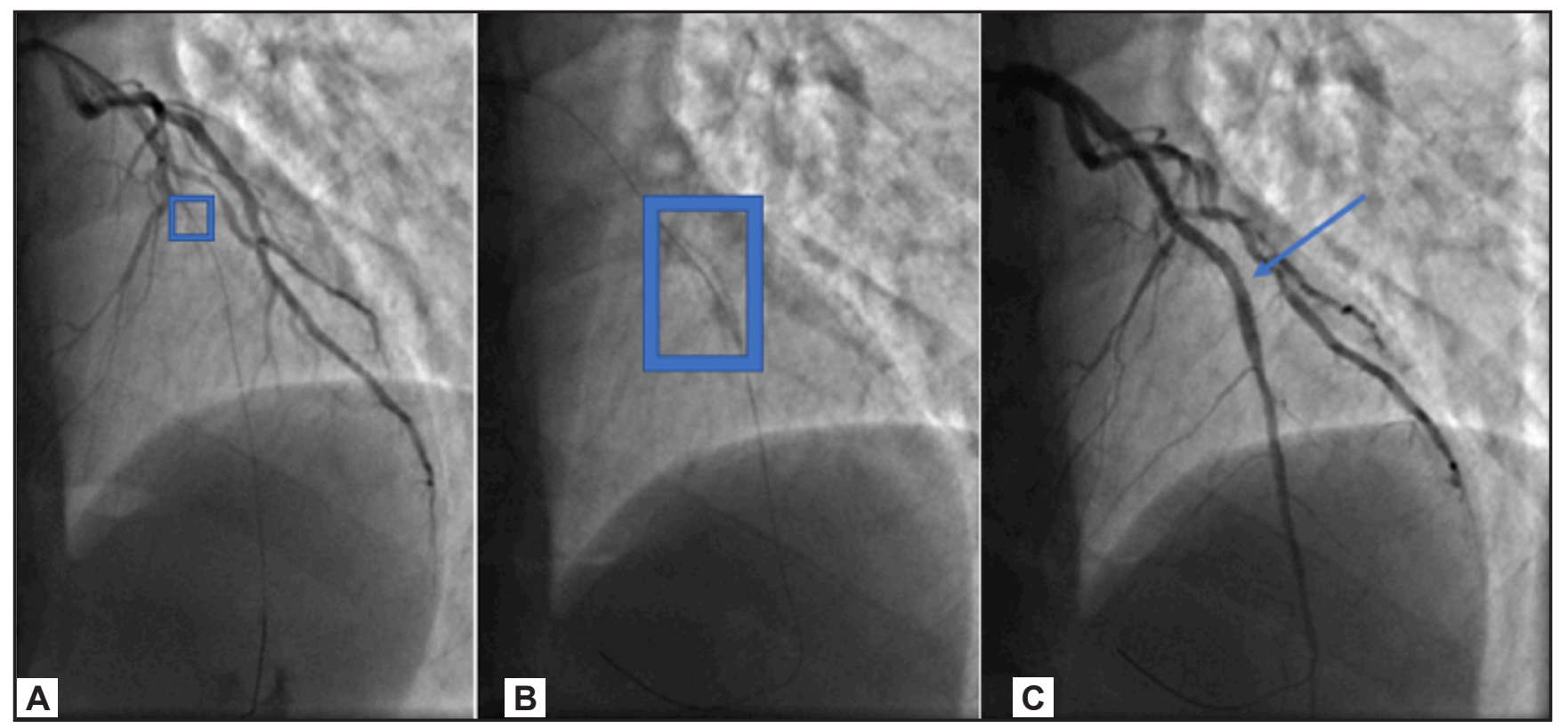

Figura 2. Angioplastía primaria encontrando obstrucción del $100 \%$ de la arteria descendente anterior en el tercio proximal (Figura $2 \mathrm{~A})$, lugar donde se realiza implante de dos stent medicados (Figuras $2 \mathrm{~B}-2 \mathrm{C}$ ). 


\section{Discusión}

Todos los pacientes con dolor en el pecho después de la ingestión de cocaína tienen que tener de inmediato un electrocardiograma (ECG) de 12 derivaciones y una radiografía de tórax, dado que aparte del síndrome coronario agudo se tiene como diagnóstico diferencial la disección aórtica, presente en alguna series de casos donde un porcentaje alto de pacientes ( $>20 \%$ ) tenían antecedente reciente de consumo y disección. $(10,11)$.

En cuanto al enfoque del dolor torácico, la lectura de electrocardiograma e interpretación de biomarcadores cardiacos, se hará según lo indicado por la cuarta definición de infarto; ya que los estudios sugieren que la estratificación del riesgo y que la aproximación clínica clásica, es factible y segura utilizarla cuando se enfrenta a paciente con dolor torácico y consumo de cocaína asociada (12).

En general el infarto agudo de miocardio asociado a la cocaína ocurre con mayor frecuencia poco después del consumo, con un riesgo 24 veces mayor posterior a la primera hora de uso (13); sin embargo, se ha evidenciado que hasta con concentraciones bajas o indetectables pueden desencadenar eventos coronarios agudos, por lo que los estudios han informado que el rango se puede extender desde el primer minuto hasta los cuatro días posteriores al consumo (14).

Los efectos que tiene la cocaína sobre el sistema cardiovascular son muy diversos, teniendo varios mecanismos que pueden facilitar la isquemia miocárdica y llevar a infarto agudo de miocardio. El principal está asociado al aumento de norepinefrina circulante secundario a la inhibición de la recaptación de ésta, dando como resultado aumento de la respuesta simpática a nivel cardiovascular que desencadena mayor demanda de oxígeno del miocardio y una menor perfusión miocárdica asociado a vasoespasmo coronario $(6,7)$, si bien esta es la forma más descrita, se ha reportado que su uso crónico acelera la formación de aterosclerosis por producción de defectos en el endotelio vascular que lleva a aumento en la permeabilidad a la peroxidasa y las lipoproteínas de baja densidad (8), asociado se ha descrito trombosis por aumento en la activación y agregación plaquetaria (9) (Figura 3).

Los estudios han reportado que de 56 - $80 \%$ de los electrocardiogramas son anormales en los pacientes con dolor de pecho asociado al consumo de cocaína (9), pero no todos los cambios nos hablan de isquemia aguda o IAM. En una serie de 238 pacientes, $33 \%$ tenía ECG normales, $23 \%$ tenía cambios inespecíficos, $13 \%$ tenía un patrón de hipertrofia ventricular izquierda y $13 \%$ tenía sólo un patrón de repolarización temprana. Los cambios de ECG específicos para isquemia o infarto estuvieron presentes solo en una minoría de pacientes; sólo un pequeño porcentaje de pacientes con dolor torácico asociados con cocaína y elevación del punto J en realidad tienen un IM (9).

En cuanto al método de estratificación coronaria el médico tratante debe seguir los principios generales para

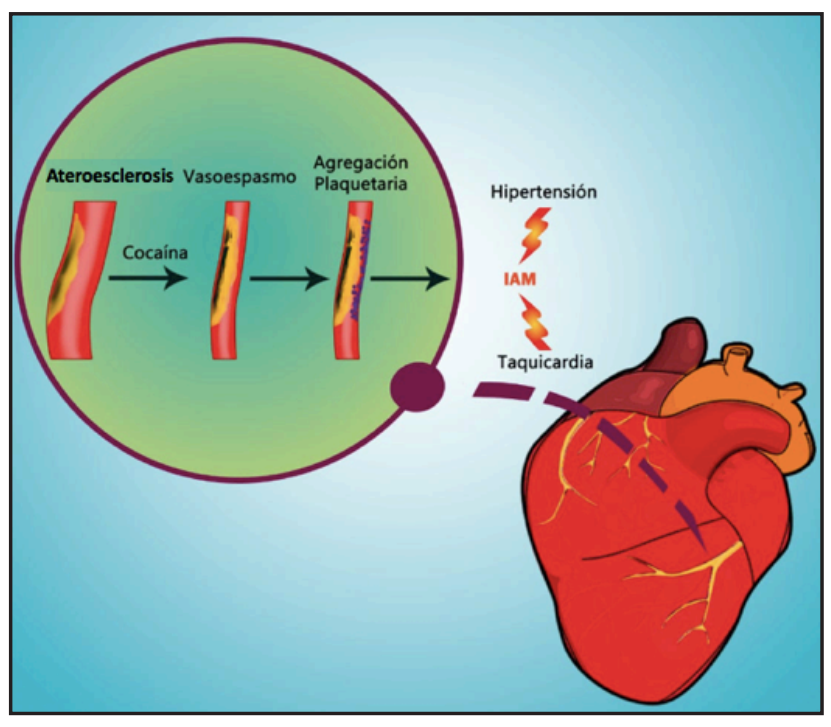

Figura 3. Fisiopatología.

la evaluación del riesgo de pacientes con posible síndrome coronario agudo en la población general, según la mejor evidencia que se tenga en el momento, a la luz de las anomalías electrocardiográficas encontradas, el resultado de biomarcadores cardiacos y según la disponibilidad de recursos con los que se cuenten.

Si un consumidor de cocaína se presenta con infarto de miocardio con elevación del segmento ST dentro del tiempo de ventana desde el inicio de dolor, se debe implementar una terapia trombolítica inmediata o una intervención coronaria percutánea, teniendo en cuenta el riesgo elevado de trombosis por aumento en la activación y agregación plaquetaria (9). Como ya se explicó anteriormente solo un pequeño porcentaje de pacientes con dolor torácico asociado con cocaína y elevación del punto $\mathrm{J}$ en realidad tienen un IM, por lo que si se tiene la disponibilidad en los tiempos estipulados para llevar a un paciente a angioplastia primaria esta es la estrategia de elección.

La importancia del reporte del caso radica en el conocimiento que el mecanismo de infarto en este grupo de pacientes no sólo es secundario a vasoespasmo, por lo tanto este paciente según lo descrito, fue llevado dentro de los tiempos estipulados a cateterismo cardiaco con angioplastia secundaria como en un paciente no consumidor.

La cocaína daña el endotelio vascular, aumenta la agregación plaquetaria y altera las vías fibrinolíticas normales, en consecuencia el IAM no solo es resultado de la respuesta simpática, por lo que el uso de ASA, inhibidores del receptor P2Y2, los antagonistas de la glucoproteína IIb / IIIa, las heparinas y las estatinas; fisiopatológicamente están claramente justificadas en este grupo de pacientes.

Los pacientes con infarto de miocardio sin elevación del segmento ST o angina inestable tienen mayor riesgo de eventos posteriores y de beneficiarse de una estratificación coronaria temprana con cateterismo cardiaco y según los 
hallazgos con revascularización miocárdica, al igual que los pacientes con SCA no relacionado al consumo de cocaína (9).

La suspensión del consumo de cocaína es el objetivo principal de la prevención secundaria, dado que la reincidencia de dolor torácico es alta entre los pacientes que persisten con el consumo (15) por lo que la disminución de riesgo de reingreso puede recaer en la implementación de programas de rehabilitación de su adicción.

El abandono del hábito de fumar, disminuir consumo de alcohol, control de comorbilidades como la hipertensión y la diabetes, terapia agresiva hipolipemiante, asociado a rehabilitación cardiovascular, son estrategias que no tienen ensayos clínicos directamente en esta población, pero son estándar para pacientes con enfermedad coronaria en general y aplican a esta condición (9).

Ya sea en el contexto de implante de stent, IAMCEST $\mathrm{o}$ alto riesgo trombótico asociado, la terapia antiagregante dual con inhibidores del receptor P2Y2 de alta intensidad como prasugrel y ticagrelor está indicada por el tiempo establecido actualmente (16), estrategia que se utilizó en el paciente descrito.

El uso crónico de bloqueadores beta deberá reservarse para aquellos con las indicaciones fuertes como disfunción sistólica ventricular izquierda, arritmias ventriculares. Su uso como prevención primaria en el contexto de uso crónico de cocaína no se ha demostrado utilidad en la reducción de eventos (9).

\section{Conclusiones}

En los últimos años se ha evidenciado un aumento en el consumo de cocaína con un inicio a muy temprana edad tanto en la población colombiana como a nivel mundial, siendo el consumo de ésta una de las principales causas de SCA en los pacientes jóvenes.

Los mecanismos fisiopatológicos son diversos y van desde la respuesta simpática aumentada como el aumento de aterosclerosis y riesgo de trombosis, con un rango de tiempo de presentación clínica posterior al consumo que es variable. El diagnóstico y manejo no se distancia mucho a las indicaciones dadas por los lineamientos internacionales para el manejo de los pacientes con SCA no relacionado al consumo de cocaína.
El manejo adecuado de la adicción toma especial relevancia en este grupo de pacientes como estrategia de prevención secundaria, sumado a las recomendaciones que aplican para los eventos coronarios no relacionados con el consumo.

\section{Referencias}

1. Gil Botero, E., Medina Ramírez, C., Rodríguez Padilla, R., Paredes Rosero, M., \& Crispín Amorocho, G. P. Reporte de drogas Colombia. 2017; 3: 224.

2. Gómez-Restrepo, C., Castro-Díaz, S., del Castillo, C. E. D., Gil, F., Villegas, C. A. A., Fagua, J., \& Araneda, J. C. Prevalencia del consumo de sustancias psicoactivas en pacientes no psiquiátricos que consultan un servicio de urgencias en Bogotá. Revista Colombiana de Psiquiatría,40(4), 709-722.

3. McCord, J., Jneid, H., Hollander, J. E., de Lemos, J. A., Cercek, B., Hsue, P., ... \& Newby, L. K. Management of cocaine-associated chest pain and myocardial infarction: a scientific statement from the American Heart Association Acute Cardiac Care Committee of the Council on Clinical Cardiology. Circulation, 117(14), 1897-1907.

4. Carrillo, X., Curós, A., Muga, R., Serra, J., Sanvisens, A., \& Bayes-Genis, A. Acute coronary syndrome and cocaine use: 8 -year prevalence and in hospital outcomes. European heart journal, 32(10), 1244-1250.

5. Singh, V., Rodriguez, A. P., Thakkar, B., Savani, G. T., Patel, N. J., Badheka, A. Hospital admissions for chest pain associated with cocaine use in the United States. The American journal of medicine, 130(6), 688-698.

6. Whitby, L. G., Hertting, G., \& Axelrod, J. Effect of cocaine on the disposition of noradrenaline labelled with tritium. Nature, 187(4737), 604

7. Muscholl E. Effect of cocaine and related drugs on the uptake of noradrenaline by heart and spleen. Br J Pharmacol Chemother. 1961; 16:352-359.

8. Kolodgie, F. D., Wilson, P. S., Mergner, W. J., \& Virmani, R. Cocaine-induced increase in the permeability function of human vascular endothelial cell monolayers. Experimental and molecular pathology, 66(2), 109-122.

9. McCord, J., Jneid, H., Hollander, J. E., de Lemos, J. A., Cercek, B., Hsue, P., ... \& Newby, L. K. Management of cocaine-associated chest pain and myocardial infarction: a scientific statement from the American Heart Association Acute Cardiac Care Committee of the Council on Clinical Cardiology. Circulation, 117(14), 1897-1907.

10. Hsue PY, Salinas CL, Bolger AF, Benowitz NL, Waters DD. Acute aortic dissection related to crack cocaine. Circulation. 2002;105: 1592-1595.

11. Eagle KA, Isselbacher EM, DeSanctis RW; International Registry for Aortic Dissection (IRAD) Investigators. Cocaine-related aortic dissection in perspective. Circulation. 2002;105:1529-1530.

12. Kushman SO, Storrow AB, Liu T, Gibler WB. Cocaine-associated chest pain in a chest pain center. Am J Cardiol. 2000;85:394-396, A10.

13. Mittleman, M. A., Mintzer, D., Maclure, M., Tofler, G. H., Sherwood, J. B., \& Muller, J.E. (1999). Triggering of myocardial infarction by cocaine. Circulation,99(21), 2737-2741.

14. Hollander JE, Hoffman RS. Cocaine-induced myocardial infarction: an analysis and review of the literature. J Emerg Med. 1992;10:169-177.

15. Hollander JE, Hoffman RS, Gennis P, Fairweather P, Feldman JA, Fish SS, DiSano MJ, Schumb DA, Dyer S. Cocaine-associated chest pain: one-year follow-up. Acad Emerg Med. 1995;2:179 -184.

16. Borja, I., Stefan, J., \& Agewall, S. Guía ESC 2017 sobre el tratamiento del infarto agudo de miocardio en pacientes con elevación del segmento ST. Revista Española de Cardiología, 1-60. 